\title{
Compactness result and its applications in integral equations
}

\author{
Mateusz Krukowski ${ }^{1}$ and Bogdan Przeradzki \\ 1,2 Technical University of Łódź, Institute of Mathematics
}

May 12, 2015

\begin{abstract}
A version of Arzelà-Ascoli theorem for $X$ being $\sigma$-locally compact Hausdorff space is proved. The result is used in proving compactness of Fredholm, Hammerstein and Urysohn operators. Two fixed point theorems, for Hammerstein and Urysohn operator, are derived on the basis of Schauder theorem.
\end{abstract}

Keywords : Arzelà-Ascoli theorem, compact operators, Fredholm, Hammerstein, Urysohn

\section{Introduction}

Compactness criteria in typical function spaces not only constitute important results describing properties of these spaces, but they also give a basic tool for investigating the existence of solutions to nonlinear equations of many kinds. The best known criterion is the Arzelà-Ascoli theorem that gives necessary and sufficient conditions for compactness in the space of continuous functions defined on a compact space $X$ and taking values in $\mathbb{R}$ or, more generally, in any finite-dimensional Banach space $E$ : a subset $K \subset C(X, E)$ should consist of equibounded and equicontinuous functions. It is easy to drop the assumption on the dimension of $E$ but then the functions from $K$ should be pointwise relatively compact, i.e. $K_{x}:=\{f(x): f \in K\}$ is relatively compact for any $x \in X$. The natural topology in $C(X, E)$ is the topology of uniform convergence given by the norm $\|f\|:=\sup _{x \in X}\|f(x)\|_{E}$.

If $X$ is not a compact space but only a locally compact one, the Arzelaà-Ascoli theorem gives a compactness criterion in the space of continuous functions $C(X, Y)$, where $Y$ is a metric space, with the topology of compact convergence, see [5] (or a compact-open topology [3]). A sequence $\left(f_{n}\right)$ tends to $f$ in $C(X, Y)$, if $\left.\left.f_{n}\right|_{F} \rightarrow f\right|_{F}$ uniformly for each compact subset $F \subset X$.

If one needs the boundedness of this limit $f$, then he should work in the space of bounded continuous functions $B C(X, Y)$ with its natural topology of uniform convergence. In the case when $Y$ is a Banach space, $B C(X, Y)$ is equipped with the same 
norm $\|f\|:=\sup \|f(x)\|$ and it is complete. Sufficient conditions for compactness of $K \subset B C(X, Y)$ were known for a long time but they were far from necessary ones and, in fact, they describe subsets of some important subspaces of functions tending to 0 at infinity (or to any other limits). In [7], one can find sufficient and necessary conditions, but in the case $X=\mathbb{R}$; an improved result is in [8].

This paper contains a general case $K \subset B C(X, Y)$, where $X$ is $\sigma$-locally compact and $Y$ is metric and complete (theorem 2). This result is applied to linear integral operators (theorem 4) and then to nonlinear integral operators of the Hammerstein (theorem [6) and Urysohn (theorem (7) type. They give fixed points, solutions of nonlinear integral equations, by using Schauder fixed point theorem. Basic facts about these operators (on compact domains) can be found in [2], [6] and [9].

\section{Notation}

In the paper, we will be mainly preoccupied with the space of bounded and continuous functions. The following definition should clarify any confusion.

Definition 1. (spaces $C(X, Y)$ and $B C(X, Y)$ )

Let $\left(X, \tau_{X}\right)$ be a topological space and $\left(Y, d_{Y}\right)$ be a metric space. $C(X, Y)$ is the space of continuous functions with the topology of compact convergence.

$B C(X, Y)$ is the space of bounded and continuous functions with the topology of uniform convergence.

Next definition is an extension of the idea found in [7. It is a key concept in theorem 2 .

Definition 2. $(B C(X, Y)$-extension condition)

Let $\left(X, \tau_{X}\right)$ be a topological space and $\left(Y, d_{Y}\right)$ be a metric space. We say that $K \subset$ $B C(X, Y)$ satisfies $B C(X, Y)$-extension condition if for all $\varepsilon>0$, there exists a compact set $D$ and $\delta>0$ such that for all $f, g \in K$

$$
d_{B C(D, Y)}(f, g) \leq \delta \Longrightarrow d_{B C(X, Y)}(f, g) \leq \varepsilon
$$

To avoid confusion, we provide the definition of $\sigma$-local compactness, which is used throughout the paper.

Definition 3. ( $\sigma$-local compactness)

We say that the topological space $\left(X, \tau_{X}\right)$ is $\sigma$-locally compact if it is locally compact and $X=\bigcup_{n=1}^{\infty} S_{n}$, where $\left(S_{n}\right)_{n=1}^{\infty}$ is a sequence of compact sets. The sequence $\left(S_{n}\right)_{n=1}^{\infty}$ is called the saturating sequence of space $X$.

\section{Compactness results}

The compactness result presented in the paper relies on the following theorem from Munkres 'Topology' [5] (theorem 47.1, page 290) : 
Theorem 1. (Arzelà-Ascoli for compact Hausdorff space)

Let $\left(X, \tau_{X}\right)$ be a locally compact Hausdorff space and $\left(Y, d_{Y}\right)$ be a metric space. Let $K \subset C(X, Y)$, where $C(X, Y)$ is equipped with the topology of compact convergence. $K$ is relatively compact iff it is pointwise relatively compact and equicontinuous at every point of $X$.

We use Munkres' theorem on the space $B C(X, Y)$ with topology of uniform (rather than compact) convergence. The improvement is due to the extension condition (definition 2).

Theorem 2. (Arzelà-Ascoli for $\sigma$-locally compact Hausdorff space)

Let $\left(X, \tau_{X}\right)$ be $\sigma$-locally compact Hausdorff space and $\left(Y, d_{Y}\right)$ be a complete metric space. The set $K \subset B C(X, Y)$ is relatively compact iff

(AA1) $K$ is pointwise relatively compact and equicontinuous at every point of $X$

(AA2) $K$ satisfies $B C(X, Y)$-extension condition

\section{Proof}

Given a sequence from $K$, by theorem 1 we choose a subsequence $\left(f_{k}\right)_{k=1}^{\infty}$, which is uniformly convergent on every compact set. For uniform convergence, it suffices to prove that the sequence satisfies Cauchy condition (due to $B C(X, Y)$ being a complete metric space).

Let $\varepsilon>0$. By condition (AA2) we know that there exists a compact set $D$ and $\delta>0$ such that for all $m, n \in \mathbb{N}$ we have

$$
\sup _{x \in D} d_{Y}\left(f_{m}(x), f_{n}(x)\right) \leq \delta \Longrightarrow \sup _{x \in X} d_{Y}\left(f_{m}(x), f_{n}(x)\right) \leq \varepsilon
$$

Since $\left(f_{k}\right)_{k=1}^{\infty}$ is uniformly convergent on $D$ we obtain that the Cauchy condition is satisfied.

$$
\text { ' }
$$

(AA1) follows from theorem 1, Assume for the sake of contradiction that (AA2) does not hold. The saturationg sequence $\left(S_{n}\right)_{n=1}^{\infty}$ can be constructed in such a way that $S_{n} \subset \operatorname{Int}\left(S_{n+1}\right)$ for every $n \in \mathbb{N}$. Hence every compact set $D$ is a subset of $S_{n}$ for large enough $n$. Negation of (AA2) means that there exists $\varepsilon>0$ such that for all $n \in \mathbb{N}$ there exist $f_{n}, g_{n} \in K$ such that

$$
\sup _{x \in S_{n}} d_{Y}\left(f_{n}(x), g_{n}(x)\right) \leq \frac{1}{n} \wedge \sup _{x \in X} d_{Y}\left(f_{n}(x), g_{n}(x)\right)>\varepsilon
$$

By theorem 1, we choose subsequences $\left(F_{n}\right)_{n=1}^{\infty} \subset\left(f_{n}\right)_{n=1}^{\infty}$ and $\left(G_{n}\right)_{n=1}^{\infty} \subset\left(g_{n}\right)_{n=1}^{\infty}$, which are convergent on compact sets to $F$ and $G$ respectively. The condition (11) implies that for all $m, n \in \mathbb{N}$ (without loss of generality $n \geq m$ ) we have 


$$
\sup _{x \in S_{m}} d_{Y}\left(F_{n}(x), G_{n}(x)\right) \leq \frac{1}{n}
$$

We conclude that $F \equiv G$, which is a contradiction due to the inequality

$$
\sup _{x \in X} d_{Y}\left(F_{n}(x), G_{n}(x)\right)>\varepsilon
$$

which holds for every $n \in \mathbb{N}$.

We observe that in theorem 2 the space $X$ can be a subset of finite-dimensional Banach space. In applications it is usually assumed to be closed. The novelty is that we do not assume $X$ to be bounded. We note that if $\left(Y, d_{Y}\right)$ possesses Heine-Borel property then pointwise relative compactness in (AA1) is equivalent to pointwise boundedness.

\section{Application to integral operators}

\subsection{Fredholm operator}

In order to avoid misapprehension, we provide the Carathéodory conditions which are used throughout the paper. Let $\left(X,\|\cdot\|_{X}\right),\left(E,\|\cdot\|_{E}\right)$ be finite-dimensional Banach spaces and $(Y, \Sigma, \mu)$ be a measure space. Let $K: X \times Y \rightarrow B(E)$, where $B(E)$ stands for the space of linear and bounded operators on $E$, satisfy the conditions

(Car1) $K(x, \cdot)$ is measurable for every $x \in X$

(Car2) $K(\cdot, y)$ is continuous for a.e. $y \in Y$

(Car3) for every $x \in X$ there exists an open neighbourhood $U_{x}$ and a function $D_{x} \in L^{1}(Y)$ such that for every $z \in U_{x}$ and a.e. $y \in Y$ we have

$$
\|K(z, y)\| \leq D_{x}(y)
$$

Observe that (Car1) implies that $\|K(x, \cdot)\|$ is measurable for every $x \in X$ and (Car2) implies $\|K(\cdot, y)\|$ is continuous for a.e. $y \in Y$. Hence the function $\Phi: X \rightarrow$ $B(E)$, defined by

$$
\Phi(x):=\int_{Y}\|K(x, y)\| d \mu(y)
$$

is continuous for every $x \in X$. Lastly, we adjoin to Carathéodory conditions (Car1)(Car3) the requirement 
(Car4) the function $K$ satisfies

$$
\sup _{x \in X} \int_{Y}\|K(x, y)\| d \mu(y)<\infty
$$

The following lemma is introduced for cosmetic reasons. The main tool used in theorem 4 is condition (K2). However, for simplicity, we provide an equivalent condition (K1).

Lemma 3. Let $\left(X,\|\cdot\|_{X}\right),\left(E,\|\cdot\|_{E}\right)$ be finite-dimensional Banach spaces and $(Y, \Sigma, \mu)$ be a measure space. Let $K: X \times Y \rightarrow B(E)$ be a function satisfying conditions (Car1)-(Car4). Then the following conditions are equivalent:

(K1) for every $\varepsilon>0$ and $v \in X,\|v\|=1$ there exist $T_{v}>0$ and $L_{v} \in$ $L^{1}(Y, B(E))$ such that for every $t \geq T_{v}$ we have

$$
\int_{Y}\left\|K(t v, y)-L_{v}(y)\right\| d \mu(y) \leq \varepsilon
$$

and moreover $\sup _{\|v\|=1} T_{v}<\infty$

(K2) for every $\varepsilon>0$ and $v \in X,\|v\|=1$ there exists $T_{v}>0$ such that for every $t, s \geq T_{v}$ we have

$$
\int_{Y}\|K(t v, y)-K(s v, y)\| d \mu(y) \leq \varepsilon
$$

and moreover $\sup _{\|v\|=1} T_{v}<\infty$

\section{Proof}

$(\mathrm{K} 1) \Longrightarrow(\mathrm{K} 2)$

It suffices to note that the triangle inequality

$$
\|K(t v, y)-K(s v, y)\| \leq\left\|K(t v, y)-L_{v}(y)\right\|+\left\|L_{v}(y)-K(s v, y)\right\|
$$

holds for every $t, s \geq 0, v \in X,\|v\|=1$ and a.e. $y \in Y$.

$(\mathrm{K} 1) \Longleftarrow(\mathrm{K} 2)$

Recognizing (K2) as the Cauchy condition, we obtain the existence of $L_{v} \in L^{1}(Y)$ by completeness of the space $L^{1}(Y, B(E))$.

Remark 1. The implication (K1) $\Longleftarrow(\mathbf{K 2})$ in the proof of lemma 3 does not explicitly construct the limit. In order to obtain a constructive proof, assume additionally 
- there exists a function $D \in L^{1}(Y)$ such that for every $x \in X$ and a.e. $y \in Y$, we have

$$
\|K(x, y)\| \leq D(y)
$$

- for every $v \in X,\|v\|=1$ and a.e. $y \in Y$ the $\operatorname{limit}_{\lim } \rightarrow \infty K(s v, y)$ exists

Then the limit function in (K1) is given by

$$
L_{v}(y)=\lim _{s \rightarrow \infty} K(s v, y)
$$

because we can interchange integral sign with the limit in (K2) due to the Lebesgue dominated convergence theorem.

Theorem 4. Let $\left(X,\|\cdot\|_{X}\right),\left(E,\|\cdot\|_{E}\right)$ be finite-dimensional Banach spaces and $(Y, \Sigma, \mu)$ be a measure space. Let $K: X \times Y \rightarrow B(E)$ satisfy the condtions (Car1)(Car4) and (K1). Then the Fredholm operator $\mathcal{F}: L^{\infty}(Y, E) \rightarrow B C(X, E)$ defined by

$$
(\mathcal{F} f)(x):=\int_{Y} K(x, y) f(y) d \mu(y)
$$

is linear and compact.

\section{Proof}

We check that the operator is well-defined. The following estimate proves boundedness $\left(f \in L^{\infty}(Y, E)\right.$ is fixed) of the Fredholm operator

$$
\sup _{x \in X}\|(\mathcal{F} f)(x)\| \leq\|f\| \sup _{x \in X} \int_{Y}\|K(x, y)\| d \mu(y)
$$

For the rest of the proof we fix $\varepsilon>0$. We prove continuity of the function $(T f)$ at $x_{*} \in$ $X$, where we assume $f \not \equiv 0$ (otherwise continuity is trivial). By conditions (Car1)(Car4) we know that there exists $\delta>0$ such that for every $x \in X,\left\|x-x_{*}\right\|<\delta$ we have

$$
\int_{Y}\left\|K(x, y)-K\left(x_{*}, y\right)\right\| d \mu(y) \leq \frac{\varepsilon}{\|f\|}
$$

Hence, for every $x \in X,\left\|x-x_{*}\right\|<\delta$ we obtain the following inequality

$$
\begin{gathered}
\left\|(\mathcal{F} f)(x)-(\mathcal{F} f)\left(x_{*}\right)\right\|=\left\|\int_{Y} K(x, y) f(y) d \mu(y)-\int_{Y} K\left(x_{*}, y\right) f(y) d \mu(y)\right\| \\
\leq\|f\| \int_{Y}\left\|K(x, y)-K\left(x_{*}, y\right)\right\| d \mu(y) \leq \varepsilon
\end{gathered}
$$


which proves the continuity of $\mathcal{F} f$.

In order to prove compactness (using theorem 2), denote

$$
B:=\left\{f \in L^{\infty}(Y, E):\|f\|<1\right\}
$$

$\mathcal{F}(B)$ is bounded (in $B C(X, E)$ ) due to the estimate (2). The equicontinuity of $\mathcal{F}(B)$ at $x_{*}$ follows from the estimate (3). Hence the condition (AA1) is satisfied.

It remains to prove that $\mathcal{F}(B)$ satisfies $B C(X, E)$-extension condition, i.e. there exist $T>0$ and $\delta>0$ such that for every $f, g \in B$ we have

$$
\sup _{\|x\| \leq T}\left\|\int_{Y} K(x, y)(f(y)-g(y)) d y\right\| \leq \delta \Longrightarrow \sup _{x \in X}\left\|\int_{Y} K(x, y)(f(y)-g(y)) d y\right\| \leq \varepsilon
$$

By condition (K1) we know that there exists $T>0$ such that for every $v \in X,\|v\|=$ 1 and every $t, s>T$ we have

$$
\int_{Y}\|K(t v, y)-K(s v, y)\| d \mu(y) \leq \frac{\varepsilon}{4}
$$

For arbitrary $f, g \in B$ denote $h:=f-g$. For every $v \in X,\|v\|=1$ and every $t \geq T$ we have

$$
\begin{gathered}
\|\| \int_{Y} K(t v, y) h(y) d \mu(y)\|-\| \int_{Y} K(T v, y) h(y) d \mu(y)\|\| \\
\leq\left\|\int_{Y} K(t v, y) h(y)-K(T v, y) h(y) d \mu(y)\right\| \leq \int_{Y}\|K(t v, y)-K(T v, y)\|\|h(y)\| d \mu(y) \leq \frac{\varepsilon}{2}
\end{gathered}
$$

By estimate (5), for every $v \in X,\|v\|=1$ and $t \geq T$ we obtain

$$
\left\|\int_{Y} K(t v, y) h(y) d \mu(y)\right\| \leq \frac{\varepsilon}{2}+\sup _{\|x\| \leq T}\left\|\int_{Y} K(x, y) h(y) d \mu(y)\right\|
$$

The estimate (6) gives

$$
\sup _{\|x\|>T}\left\|\int_{Y} K(x, y) h(y) d \mu(y)\right\| \leq \frac{\varepsilon}{2}+\sup _{\|x\| \leq T}\left\|\int_{Y} K(x, y) h(y) d \mu(y)\right\|
$$

Hence it suffices to take $\delta=\frac{\varepsilon}{4}$ for the implication (4) to be satisfied. 
Corollary 5. Let $\left(X,\|\cdot\|_{X}\right)$ be a finite-dimensional Banach space and $\left(Y, \tau_{Y}\right)$ be a topological space with Borel measure $\mu$. Let $K \in B C(X \times Y, \mathbb{C})$ satisfy (Car3)$(\mathbf{C a r} 4)$ and $(\mathbf{K} 1)$. Then the Fredholm operator $\mathcal{F}: B C(Y, \mathbb{C}) \rightarrow B C(X, \mathbb{C})$ is linear and compact.

In order to present an application of corollary 5 , we consider a well-known example of non-compact integral operator $\mathcal{P}: B C(\mathbb{R}, \mathbb{C}) \rightarrow B C(\mathbb{R}, \mathbb{C})$ defined by

$$
(\mathcal{P} f)(x)=\int_{\mathbb{R}} e^{-|x-y|} f(y) d y
$$

After small modifications, we are able to obtain a positive result.

Example 1. Let $n \in \mathbb{N}$ and $g: \mathbb{R}^{n} \rightarrow \mathbb{R}^{n}$ be a continuous function such that for every $\varepsilon>0$ and $v \in X,\|v\|<1$ there exist $T_{v}>0$ and $g_{v} \in \mathbb{R}^{n}$ such that for every $t \geq T_{v}$ we have $\left\|g(t v)-g_{v}\right\| \leq \varepsilon$ and moreover $\sup _{\|v\|=1} T_{v}<\infty$. Then the integral operator $\mathcal{P}: L^{\infty}\left(\mathbb{R}^{n}, \mathbb{C}\right) \rightarrow B C\left(\mathbb{R}^{n}, \mathbb{C}\right)$ defined by

$$
(\mathcal{P} f)(x)=\int_{\mathbb{R}^{n}} e^{-\|g(x)-y\|} f(y) d y
$$

is linear and compact.

\section{Solution}

(Car3)-(Car4) follows from the rapid decay of exponential function and the equality

$$
\int_{\mathbb{R}^{n}} e^{\|g(x)-y\|} d y=\int_{\mathbb{R}^{n}} e^{-\|y\|} d y
$$

Obviously $e^{-\left\|g_{v}-\cdot\right\|} \in L^{1}\left(\mathbb{R}^{n}\right)$ for every $v \in X,\|v\|=1$. In order to prove (K1) we note the estimate

$$
\left|e^{-a}-e^{-b}\right| \leq e^{-b}\left|e^{-(a-b)}-1\right| \leq e^{-b}\left(e^{|a-b|}-1\right)
$$

which holds for $a, b \geq 0$. We set $a=\|g(t v)-y\|, b=\left\|g_{v}-y\right\|$ and integrate over $\mathbb{R}^{n}$. For every $v \in X,\|v\|=1$ we obtain

$$
\begin{gathered}
\int_{\mathbb{R}^{n}} \mid e^{-\|g(t v)-y\|}-e^{-\left\|g_{v}-y\right\| \mid} d y \leq\left(e^{\left\|g(t v)-g_{v}\right\|}-1\right) \int_{\mathbb{R}^{n}} e^{-\left\|g_{v}-y\right\|} d y \\
=\left(e^{\left\|g(t v)-g_{v}\right\|}-1\right) \int_{\mathbb{R}^{n}} e^{-\|y\|} d y
\end{gathered}
$$

Fix $\varepsilon>0$ and choose $T>0$ such that for every $v \in X,\|v\|=1$ and $t \geq T$ we have

$$
\left(e^{\left\|g(t v)-g_{v}\right\|}-1\right) \int_{\mathbb{R}^{n}} e^{-\|y\|} d y \leq \varepsilon
$$

By estimate (17), we are done. 
Example 2. Let $n \in \mathbb{N}$ and $K: \mathbb{R}^{n} \times \mathbb{R}^{n} \rightarrow \mathbb{C}$ satisfy (Car1)-(Car4) and (K1). Then the Volterra operator $\mathcal{V}: L^{\infty}\left(\mathbb{R}^{n}, \mathbb{C}\right) \rightarrow B C\left(\mathbb{R}^{n}, \mathbb{C}\right)$ defined by

$$
(\mathcal{V} f)(x):=\int_{-\infty}^{x_{n}} \ldots \int_{-\infty}^{x_{1}} K(x, y) f(y) d y_{1} \ldots d y_{n}
$$

where $x=\left(x_{1}, \ldots, x_{n}\right)$ and $y=\left(y_{1}, \ldots, y_{n}\right)$, is linear and compact.

\section{Solution}

We approximate the Volterra operator $\mathcal{V}$ with compact Fredholm operators $\left(\mathcal{F}_{m}\right)_{m \in \mathbb{N}}$. For every $x, y \in \mathbb{R}^{n}$ let

$$
K_{m}(x, y)=K(x, y) \prod_{k=1}^{n}\left(\mathbb{1}_{\left(-\infty, x_{k}\right]}\left(y_{k}\right)+\left(-m y_{k}+m x_{k}+1\right) \mathbb{1}_{\left(x_{k}, x_{k}+\frac{1}{m}\right)}\left(y_{k}\right)\right)
$$

Kernels $K_{m}$ satisfy (Car1)-(Car4) and (K1) and hence for every $f \in L^{\infty}\left(\mathbb{R}^{n}, \mathbb{C}\right),\|f\|<$ 1 we have the estimate

$$
\begin{aligned}
\left\|\mathcal{F}_{m} f-\mathcal{V} f\right\| \leq \sup _{x \in \mathbb{R}^{n}} & \int_{x_{n}}^{x_{n}+\frac{1}{m}} \ldots \int_{x_{1}}^{x_{1}+\frac{1}{m}}|K(x, y)| \prod_{k=1}^{n}\left|-m y_{k}+m x_{k}+1\right| d y_{1} \ldots d y_{n} \\
& \sup _{x \in \mathbb{R}^{n}} \int_{x_{n}}^{x_{n}+\frac{1}{m}} \ldots \int_{x_{1}}^{x_{1}+\frac{1}{m}}|K(x, y)| d y_{1} \ldots d y_{n}
\end{aligned}
$$

The convergence $\mathcal{F}_{m} \rightarrow \mathcal{V}$ in the operator norm follows from proposition 3.3.9 (page 112) in [1].

Remark 2. We could not consider the kernel

$$
x \mapsto K(x, y) \prod_{k=1}^{n} \mathbb{1}_{\left(-\infty, x_{k}\right]}\left(y_{k}\right)
$$

in place of (8), beacuse it violates (Car2).

\subsection{Hammerstein and Urysohn operator}

Theorem 6. Let $\left(X,\|\cdot\|_{X}\right),\left(E,\|\cdot\|_{E}\right)$ be finite-dimensional Banach spaces, $(Y, \Sigma, \mu)$ be a measure space and $K: X \times Y \rightarrow B(E)$ satisfy the condtions (Car1)-(Car4) and (K1). Moreover, let $F: Y \times E \rightarrow E$ satisfy

(F1) for every $z \in E$ the function $F(\cdot, z)$ is measurable 
(F2) for every $z \in E$ and $\varepsilon>0$ there exists $\delta>0$ such that for a.e. $y \in Y$ and $w \in E$ we have

$$
\|w-z\| \leq \delta \Longrightarrow\|F(y, w)-F(y, z)\| \leq \varepsilon
$$

(F3) there exists a nondecreasing continuous function $\phi:[0, \infty) \rightarrow \mathbb{R}$ such that for a.e. $y \in Y, z \in E$ we have

$$
\|F(y, z)\| \leq \phi(\|z\|)
$$

Then the Hammerstein operator $\mathcal{H}: L^{\infty}(Y, E) \rightarrow B C(X, E)$ defined by

$$
(\mathcal{H} f)(x):=\int_{Y} K(x, y) F(y, f(y)) d \mu(y)
$$

is compact. Moreover, if $X=Y$ and there is $t_{*} \in(0, \infty)$ satisfying

$$
\sup _{x \in X} \int_{Y}\|K(x, y)\| d \mu(y) \phi\left(t_{*}\right)=t_{*}
$$

then the Hammerstein operator $\mathcal{H}: B C(X, E) \rightarrow B C(X, E)$ has a fixed point.

\section{Proof}

Observe that the Hammerstein operator $\mathcal{H}$ is the composition of the Fredholm operator $\mathcal{F}: L^{\infty}(Y, E) \rightarrow B C(X, E)$ from theorem 4 and Nemytskii operator $\mathcal{N}$ : $L^{\infty}(Y, E) \rightarrow L^{\infty}(Y, E)$, defined by

$$
(\mathcal{N} f)(y):=F(y, f(y))
$$

The fact that Nemytskii operator maps measurable functions to measurable functions follows from (F1)-(F2) and the argument in [4] (page 349). By (F3), Nemytskii operator maps bounded sets to bounded sets (in the sense of $L^{\infty}(Y, E)$-norm). Hence, the operator $\mathcal{H}$ maps bounded sets to compact sets. Furthermore, (F2) implies the continuity of $\mathcal{N}$ and thus the composition $\mathcal{H}=\mathcal{F} \circ \mathcal{N}$ is also continuous, which proves that the Hammerstein operator is compact.

Assume now, that $X=Y$ and that $\mathcal{H}: B C(X, E) \rightarrow B C(X, E)$. Let $R>0$ and denote

$$
B_{R}:=\{f \in B C(X, E):\|f\| \leq R\}
$$

For every $f \in B_{R}$ we have

$$
\begin{gathered}
\sup _{x \in X}\left\|\int_{X} K(x, y) F(y, f(y)) d \mu(y)\right\| \leq \sup _{x \in X} \int_{X}\|K(x, y)\| \phi(\|f(y)\|) d \mu(y) \\
\leq \sup _{x \in X} \int_{X}\|K(x, y)\| d \mu(y) \phi(R)
\end{gathered}
$$


(F3) together with (10) implies that $\mathcal{H}\left(B_{R_{*}}\right) \subset B_{R_{*}}$ for some $R_{*}>0$. Due to Schauder fixed point theorem, we are done.

We shall show an example of applications of the compactness criterion (Theorem 2) to Urysohn integral equations, where the $B C$-extension condition is obtained in another way. Let $\mathbb{R}_{+}:=[0, \infty), E$ be a finite dimensional Banach space and let $K$ : $\mathbb{R}_{+} \times \mathbb{R}_{+} \times E \rightarrow E$ be a continuous map which is uniformly continuous with respect to (w.r.t.) the first variable. Define an integral operator $\mathcal{U}: B C\left(\mathbb{R}_{+}, E\right) \rightarrow B C\left(\mathbb{R}_{+}, E\right)$ by the formula

$$
(\mathcal{U} f)(x):=\int_{0}^{\infty} K(x, y, f(y)) d y .
$$

It is well defined if, for any $M>0$,

$$
K_{M}:=\sup _{x \in \mathbb{R}_{+}} \int_{0}^{\infty} \sup _{|u| \leq M}|K(x, y, u)| d y<\infty .
$$

Notice that the uniform continuity of $K$ w.r.t. $x$ is too strong although sufficient condition for $\mathcal{U} f$ being continuous but we shall use this assumption in the future. The main assumption (which means that, in a sense, $K$ does not depend on $u$ asymptotically) is the following

(B) there exists a function $b \in B C\left(\mathbb{R}_{+}^{2}, E\right)$ such that for any $\varepsilon>0$ and $M>0$, there is $T>0$ with the property

$$
\int_{T}^{\infty}|K(x, y, u)-b(x, y)| d y<\varepsilon
$$

for $x \in \mathbb{R}_{+},|u| \leq M$.

Theorem 7. Under the above assumptions the Urysohn operator $\mathcal{U}$ is compact. If, moreover,

$$
\limsup _{M \rightarrow \infty} \frac{K_{M}}{M}<1
$$

then $\mathcal{U}$ has a fixed point.

\section{Proof}

First, we prove the equicontinuity of $\mathcal{U} f$ for $\|f\| \leq M$. Take $\varepsilon>0$ and $T>0$ from condition (B). Since $K$ is uniformly continuous w.r.t. $x$, one can find $\delta>0$ such that

$$
\left|K\left(x_{1}, y, u\right)-K\left(x_{2}, y, u\right)\right|<\frac{\varepsilon}{T}, \quad \text { for } \quad\left|x_{1}-x_{2}\right|<\delta, \quad y \in[0, T], \quad|u| \leq M .
$$

Then, for $\|f\| \leq M$ we have 


$$
\begin{aligned}
\mid(\mathcal{U} f)\left(x_{1}\right) & -(\mathcal{U} f)\left(x_{2}\right)\left|\leq \int_{0}^{T}\right| K\left(x_{1}, y, f(y)\right)-K\left(x_{2}, y, f(y)\right) \mid d y \\
& +\int_{T}^{\infty} \sum_{i=1}^{2}\left|K\left(x_{i}, y, f(y)\right)-b\left(x_{i}, y\right)\right| d y<3 \varepsilon .
\end{aligned}
$$

Now, let $f_{1}, f_{2} \in B C\left(\mathbb{R}_{+}, E\right)$ be two functions with norms $\leq M$. Taking $T>0$ by condition (B) and then $\delta>0$ such that, for $x, y \in[0, T],\left|u_{1}\right|,\left|u_{2}\right| \leq M$ and $\left|u_{1}-u_{2}\right|<\delta$,

$$
\left|K\left(x, y, u_{1}\right)-K\left(x, y, u_{2}\right)\right|<\frac{\varepsilon}{T}
$$

we get

$$
\begin{aligned}
\mid\left(\mathcal{U} f_{1}\right)(x) & -\left(\mathcal{U} f_{2}\right)(x)\left|\leq \int_{0}^{T}\right| K\left(x, y, f_{1}(y)\right)-K\left(x, y, f_{2}(y)\right) \mid d y \\
& +\int_{T}^{\infty} \sum_{i=1}^{2}\left|K\left(x, y, f_{i}(y)\right)-b(x, y)\right| d y<3 \varepsilon .
\end{aligned}
$$

provided that $\sup \left\{\left|f_{1}(y)-f_{2}(y)\right|: y \in[0, T]\right\} \leq \delta$. It follows that $\mathcal{U}$ is continuous operator and it satisfies the $B C$-extension condition. Thus, the image of the set of functions with norms bounded by $M$ satisfies the assumptions of Theorem 2 and $\mathcal{U}$ is compact.

If condition (12) holds, then there exists $R>0$ such that $K_{R} \leq R$. It follows that the ball $\bar{B}(0, R)$ is mapped into itself by $\mathcal{U}$ and a fixed point is given due to the Schauder fixed point theorem.

\section{References}

[1] Benedetto J.J., Czaja W. : Integration and Modern Analysis, Birkhauser (2009)

[2] Corduneanu C. : Integral Equations and Applications, Cambridge University Press (1991)

[3] Engelking R. : General Topology, PWN Polish Scientific Publishers, Warsaw (1997)

[4] Krasnosielskii M.A., Zabreiko P.P., Pustylnik E.I., Sbolevskii P.E. : Integral operators in spaces of summable functions, Noordhoff International Publishing, Leyden (1976)

[5] Munkres J. : Topology, Prentice Hall, Inc. (2000)

[6] Porter D., Stirling D. : Integral equations. A practical treatmment, from spectral theory to applications, Cambridge University Press (1990) 
[7] Przeradzki B. : The existence of bounded solutions for differential equations in Hilbert spaces, Annales Polonici Mathematici, LVI.2 (1992)

[8] Stańczy R. : Hammerstein equation with an integral over noncompact domain, Annales Polonici Mathematici, 69 (1998)

[9] Zemyan S.M. : The Classical Theory of Integral Equations, Springer Science+Business, LLC (2012)

Krukowski M. (corresponding author), TEChNicAl Univeristy of ŁóDŹ, Institute of MathEMATiCs, Wólczańska 215, 90-924 Łódź, Poland

E-mail address : krukowski.mateusz13@gmail.com

Przeradzki B., Technical Univeristy of Łódź, Institute of Mathematics, Wólczańska 215, 90-924 ŁóDŹ, POLAND

E-mail address : przeradz@p.lodz.pl 